\title{
Study on the Antilipidemic Activity of Artemisia Annua Aqueous Extract
}

\author{
Ali Tao, Xuehua Feng, Zurong Song, Bo Hu, Huan You, Ruilin Wang \\ College of Pharmacy, Anhui Xinhua University, Hefei, Anhui Province, 230088, China
}

\begin{abstract}
Artemisia annua is an annual herb, which is a traditional Chinese medicine in China. The high water soluble artemisinin derivative extracted from Artemisia annua is Artemisia annua water extract. In this experiment, the modeling method is used, and the Artemisia annua water extract is used alone or in combination with other lipid-lowering drugs. Finally, the results showed that the effect of the combination of drugs was better than that of Artemisia annua water extract alone, and the effect of the drug ratio of 1:1 was the best. It can significantly reduce the content of triglyceride in serum and cholesterol, increase the ratio of $\mathrm{H} / \mathrm{L}$, and show a good synergistic effect.
\end{abstract}

\section{Introduction}

Artemisia annua is the dry aboveground part of the dicotyledonous plant Artemisia annua. It is picked in the autumn when the flowers are in full bloom to remove the old stems and avoid direct sunlight. It is put in a ventilated place and dry[1,2]. Artemisia annua belongs to the traditional Chinese medicine, which has the functions of eliminating pathogenic heat from the blood, relieving from summer heat and treating malaria[3]. Artemisia annua is mainly composed of sesquiterpenoids, and artemisinin extracted from Artemisia annua is [4] most famous for its antimalarial effect. Artemisinin and its derivatives have the effects of anti fibrosis[5], anti tuberculosis[6] and Protecting Nonalcoholic fatty liver[7]. Habibi team[8] found that Artemisia annua seed essential oil has a very high anti Haemophilus effect on Lactococcus. In addition,Artemisinin can better change the transformation of lymphocytes and the immune function of cells.

Artemisia annua water extract is the decoction extract of Artemisia annua. The high water soluble artemisinin derivative extracted from Artemisia annua is Artemisia annua water extract.Artemisia annua water extract has anticancer, antibacterial, malaria, antiviral, antitumor and other pharmacological effects. The antipyretic and anti-inflammatory effects of Artemisia annua aqueous extract are manifested in its ethyl acetate and n-butanol parts, which have obvious antipyretic and heat-resistant effects. The aqueous extract of Artemisia annua can inhibit a kind of DNA virus, HIV, and other viruses[9,10]. The anti-tumor mechanism of Artemisia annua water extract mainly includes the production of toxic free radicals by peroxy bridge, cell cycle arrest, induction of apoptosis and inhibition of tumor angiogenesis[11].In recent years, the incidence of hyperlipidemia has increased significantly[12,13]. Therefore, the study on the antilipidemic activity of Artemisia annua water extract is of great significance for the development of new antilipidemic drugs.

\section{Experimental section}

\subsection{Chemistry Materials and apparatuses.}

Male SD rats: weight 150-195g, Shanghai slake experimental animal Co,Ltd, animal Certificate No: SCXK, 2007-0005.Artemisia annua water extract: Chengdu Oukang Pharmaceutical Co,Ltd, batch No: CK018329.Ursolic acid: Xi'an Xiaocao Plant Technology Co, Ltd, batch No: XC091225.Rosuvastatin: Wuxi Shengfu Pharmaceutical Co, Ltd, batch No: 124F027116.Biochemical analyzer: Shanghai Cham Technology Co,Ltd,model: BW0237

Electronic balance: Shanghai Precision Scientific Instrument Co, Ltd, model: JA1003

Centrifuge: Hunan Kaida Scientific Instrument Co, Ltd, model: KH19A

corresponding author's e-mail: taoali84@163.com 


\subsection{Methods.}

\subsubsection{Dose Setting}

The experiment was divided into 11 groups according to different dosage ratio, and the dosage grade of experimental group was set. $30 \mathrm{mg} / \mathrm{kg}$ was the large dosage, $15 \mathrm{mg} / \mathrm{kg}$ was the average dosage, and $10 \mathrm{mg} / \mathrm{kg}$ was the small dosage.

\subsubsection{Experimental Process}

According to the experimental groups, rats were infused with different doses of different drugs. The experimental period was one month. Rats were weighed and recorded every week during the experiment. At the 26th day of the experiment, rats were anesthetized with $25 \%$ barbital sodium $(0.5 / 100 \mathrm{~g})$, then blood was collected from the abdominal aorta of rats, and the related indexes of blood lipid were detected by centrifuge.

\subsubsection{Molding Method and Grouping}

Using the method of making model, the rats were fed with high-fat feed (high-fat feed ratio: general feed $78.8 \%$, cholesterol $1 \%$, bile salt $0.2 \%$, egg yolk powder $10 \%$, lard $10 \%$ ) to induce the model of lipid metabolism disorder, and then the rats were given drugs, and finally the blood lipid level in the rats was detected.

Then according to the different dose grouping study $t$ hrough the comparison of relevant experimental data to $d$ raw a conclusion.

100 male SD rats weighing 150-195g were fed normally for 7 days, and then randomly divided into 11 groups according to the animal weight, two to three rats in each group.
The research groups of the experiment include the common group9, the experimental group 10 (1\%polysorbate), the control group11(rosuvastatin group $10 \mathrm{mg} / \mathrm{kg}$ ) and the eight receiving groups, the eight groups receiving the medicine include mixed high-dose artemisia water extract group1 $(30 \mathrm{mg} / \mathrm{kg})$, mixed average dose artemisia water extract group2 $(15 \mathrm{mg} / \mathrm{kg})$, mixed high-dose ursolic acid group3 $(30 \mathrm{mg} / \mathrm{kg})$, mixed average dose ursolic acid group4 $(15 \mathrm{mg} / \mathrm{kg})$, mixed high-dose group5 (artemisia water extract $30 \mathrm{mg} / \mathrm{kg}+$ ursolic acid $30 \mathrm{mg} / \mathrm{kg}$ ), mixed average dose group6 (artemisia water extract $10 \mathrm{mg} / \mathrm{kg}+$ ursolic acid $15 \mathrm{mg} / \mathrm{kg}$ ), mixed small dose group7 (artemisia water extract $15 \mathrm{mg} / \mathrm{kg}+$ ursolic acid $30 \mathrm{mg} / \mathrm{kg}$ ), compound control group8 (artemisia water extract $10 \mathrm{mg} / \mathrm{kg}+$ rosuvastatin $10 \mathrm{mg} / \mathrm{kg}$ ) .

\section{Results and discussion}

\subsection{The Rnfluence of Plasma Cholesterol and Triglyceride}

The changes of plasma $\mathrm{CHO}$ and TG in rats before and after the preventive treatment of Artemisia annua water extract, ursolic acid and rosuvastatin are shown in Table 1.

According to the results of the above table, the total cholesterol in the plasma of the high-fat model rats was significantly higher than that of the common group after 3 weeks of treatment, and the cholesterol and laurel lipid in the plasma of the high-fat model rats were significantly lower than that of the experimental group. The best group was the mixed high-dose group, the mixed average dose group, the second group was the mixed low-dose group, which were better than those of Artemisia annua water extract, ursolic acid and rosuvastatin in the positive control group.

Table 1 Changes of blood lipid level in rats before and after administration

\begin{tabular}{ccl}
\hline Group number & CHO & \multicolumn{1}{c}{ TG } \\
\hline 1 & $6.21 \pm 2.32^{2)}$ & $0.68 \pm 0.48$ \\
2 & $6.05 \pm 2.16$ & $0.52 \pm 0.32^{1^{\prime}}$ \\
3 & $5.67 \pm 1.58^{1^{\prime}}$ & $0.70 \pm 0.29^{2}$ \\
4 & $6.28 \pm 2.18$ & $0.56 \pm 0.31$ \\
5 & $4.18 \pm 1.45^{2)}$ & $0.58 \pm 0.17^{1}$ \\
6 & $5.27 \pm 2.02$ & $0.73 \pm 0.28$ \\
7 & $5.56 \pm 2.19^{1)}$ & $0.63 \pm 0.36^{2}$ \\
8 & $5.78 \pm 1.32$ & $0.48 \pm 0.37^{1}$ \\
9 & $2.57 \pm 2.23$ & $1.38 \pm 0.58$ \\
10 & $7.68 \pm 3.53^{4)}$ & $1.77 \pm 0.46$ \\
11 & $5.89 \pm 2.63^{1)}$ & $0.54 \pm 0.32^{1)}$
\end{tabular}

Note: 1) $\mathrm{P}<0.05$, 2) $\mathrm{P}<0.01$, compared with the experimental group, 3) $\mathrm{P}<0.05$, 4) $\mathrm{P}<0.01$, compared with the common group. 


\subsection{Effects of High and Low Density Lipoprotein Cholesterol Levels}

The changes of plasma HDL-C, LDL-C and ratio in rats are shown in Table 2.

According to the results of the above table, there was no significant change in HDL-C compared with the common group, but LDL-C increased significantly compared with the common group, so the $\mathrm{H} / \mathrm{L}$ ratio decreased significantly. Compared with the experimental group $(\mathrm{P}<0.01)$, HDL-C in the mixed high dose group increased significantly, followed by HDL-C in the mixed average dose group, followed by the mixed low dose group; compared with the experimental group $(\mathrm{P}<0.05)$, LDL-C in the mixed high dose group decreased significantly, followed by LDL-C in the mixed average dose group, and $\mathrm{H} / \mathrm{L}$ value also increased significantly.

Table 2 Effect of high and low density lipoprotein cholesterol levels and ratios

\begin{tabular}{cccc}
\hline Group number & HDL-C & LDL-C & H/L \\
\hline 1 & $0.68 \pm 0.07$ & $2.18 \pm 0.86$ & $0.31 \pm 0.22$ \\
2 & $0.66 \pm 0.09$ & $2.08 \pm 0.67$ & $0.36 \pm 0.28^{1^{\prime}}$ \\
3 & $0.63 \pm 0.02$ & $2.25 \pm 0.73$ & $0.33 \pm 0.20^{1^{\prime}}$ \\
4 & $0.60 \pm 0.10$ & $1.98 \pm 0.75$ & $0.34 \pm 0.21$ \\
5 & $0.69 \pm 0.13$ & $1.59 \pm 1.08^{1}$ & $\left.0.49 \pm 0.18^{1}\right)^{\prime}$ \\
6 & $0.64 \pm 0.09^{2}$ & $1.86 \pm 0.98^{1}$ & $0.38 \pm 0.21$ \\
7 & $0.65 \pm 0.11$ & $2.07 \pm 0.64$ & $0.30 \pm 0.22$ \\
8 & $0.67 \pm 0.08$ & $2.38 \pm 0.59^{1}$ & $0.40 \pm 0.28$ \\
9 & $0.65 \pm 0.05$ & $0.38 \pm 0.07$ & $2.56 \pm 0.40$ \\
10 & $0.62 \pm 0.12$ & $2.68 \pm 0.96^{4}$ & $\left.0.28 \pm 0.17^{4}\right)$ \\
11 & $0.68 \pm 0.15$ & $2.11 \pm 0.65$ & $0.35 \pm 0.14$ \\
\hline
\end{tabular}

which was superior to the same dose of artemisia water

\subsection{Effect of Liver Function in Rats}

The changes of AST and ALT in rats are shown in Table 3.

Table 3 Effect of liver function in rats

\begin{tabular}{ccc}
\hline $\begin{array}{c}\text { Group } \\
\text { number }\end{array}$ & AST(U/L) & ALT(U/L) \\
\hline 1 & $118.32 \pm 30.11$ & $62.71 \pm 6.53$ \\
2 & $115.27 \pm 28.35$ & $60.58 \pm 6.42$ \\
3 & $110.23 \pm 27.69$ & $59.39 \pm 6.67$ \\
4 & $120.45 \pm 19.96$ & $61.78 \pm 5.73$ \\
5 & $121.34 \pm 23.75$ & $63.98 \pm 6.32$ \\
6 & $114.82 \pm 24.43$ & $79.82 \pm 7.68$ \\
7 & $124.29 \pm 27.58$ & $65.23 \pm 6.09$ \\
8 & $118.36 \pm 30.21$ & $59.47 \pm 5.82$ \\
9 & $114.35 \pm 20.10$ & $64.32 \pm 6.08$ \\
10 & $118.47 \pm 38.16$ & $63.95 \pm 5.99$ \\
11 & $126.52 \pm 40.34$ & $81.33 \pm 7.53$ \\
\hline
\end{tabular}

The results showed that almost all drugs had no significant effect on the liver function of hyperlipidemic rats compared with the common group.

\section{Conclusion}

In the model of high-fat rats, Artemisia annua water extract has good antilipidemic activity, which can reduce the level of CHO, TG and LDL-C, or increase HDL-C, which has the effect of increasing $\mathrm{H} / \mathrm{L}$.

The best group was mixed high dose group and the second group was mixed average dose group. Therefore, the second group was used as a mixed control group, extract or ursolic acid alone and control group.Combined with Artemisia annua water extract and ursolic acid, it was found that the best ratio was $1: 1$. The mixture of Artemisia annua water extract and other antilipidemic drugs will become the focus of future research and development.

\section{Acknowledgements}

This work was supported by Natural Science Research F oundation of the Department of Education of Anhui Prov ince (No.KJ2019A0874), Scientific research team of Anu i Xinhua university (kytd201908,2019xqjdx03), Demonst ration project of grassroots teaching and research Office of Anui Xinhua university ( 2019jyssfx02 ) and Innovation and entrepreneurship training program fo $r$ college students in Anhui Province(201912216191,201 912216192).

\section{References}

1. Editorial board of flora of China. Flora of China [M]. Scientific publishing, 1979,45(23):12-13(in Chinese).

2. Hu Shilin. Textual research on the herba of Artemisia annua $[\mathrm{J}]$. Asia Pacific traditional medicine, 2006,13(9)(in Chinese):18-20.

3. Zhang Huijun, Wang Shali. Effect of Artemisia annua water extract on urinary system and digestive system of rabbits $[\mathrm{J}]$. Journal of Chongqing Medical University, 2009,34 (10): 1374-1377(in Chinese). 
4. Wang $\mathrm{Xu}, \mathrm{He}$ Lin. research progress on the pharmacodynamic effect of artemisinin compounds on diseases other than malaria $[\mathrm{J}]$. Chinese Journal of new drugs, 2018,27 (19): 2258-2263(in Chinese).

5. Li Junlong, Cao Xinran, Wang Ying, etal. Effects of artemisinin on cardiac function and fibrosis in diabetic cardiomyopathy rats [J]. Shanghai Journal of traditional Chinese medicine, 2016,50(23):70-73(in Chinese).

6. Zhang Ning, Liu Yang, Li Ye, etal. Study on the solubility improvement and antituberculosis activity of new artemisinin derivatives [J]. Acta Pharma Sinica, 2019,54(01):36-40(in Chinese).

7. Pan Jinming, Xi Jianjun, Zhang Jiankang, etal. Study on the protective effect and mechanism of Artemisia annua self microemulsion on NAFLD rats [J]. Chinese Modern Applied Pharmacy,2018,35(11):1680-1683(in Chinese).

8. Habibi Z,Ghanian S,Ghasemi S,etal.Chemical composition and antibacterial activity of the volatile oil from seeds of Artemisia annua L. From Iran
[J].Nat Prod Res. 2013,27(02):198-200.

9. Ran H, Kyoungsook P, Hongyi C,etal. Artemisinin derived dimer diphenyl phosphate is an irreversible inhibitor of human cyto-megalovirus replication [J].Antimicrob Agents Chemother. 2012,56(17): 8-15.

10. Efferth T, Marschall M, Wang X,etal. Antiviral activity of ar-tesunate towards wild-type, recombinant,and ganciclovir resist-ant human cytomegaloviruses[J].Med.2002,80(14):36-45.

11. Obeid S, Alen J, Nguyen VH,et al. Artemisinin analogues as potent inhibitors of in vitro hepatitis $\mathrm{C}$ virus replication $[\mathrm{J}]$.Plos One,2013,8(12):e81-83

12. Li liming, Rao Keqin, Kong Lingzhi. Nutrition and health status of Chinese residents in 2016 [J]. Chinese Journal of epidemiology,2015,26(07):478-484(in Chinese).

13. Wang Hongyan, Wang Peichang. Investigation on blood lipid level of healthy people in Beijing [J]. Hebei medicine,2013,35(19):2990-2993(in Chinese). 